\title{
A Rare Case of Aneurysmal Bone Cyst of the Calcaneum
}

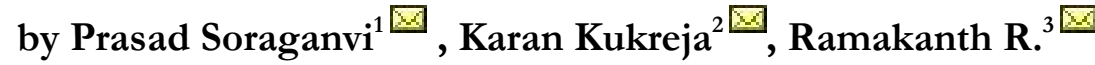

\section{The Foot and Ankle Online Journal 4 (4): 1}

Aneurysmal bone cyst $(A B C)$ is a benign solitary lesion of unknown aetiology. ABC's mainly occur in the long bones but only rarely in the bones of the feet. For example, frequency of occurrence in the foot is only 3\% compared to other bones of the body. Very few cases of ABC involving the calcaneum have been reported. We report an unusual case of $A B C$ of calcaneum in a 55 year-old male. Clinical presentation, histological diagnosis and treatment by curettage and bone grafting are described. The patient is now asymptomatic and there is no evidence of recurrence at 2 years follow-up.

Key words: ABC, calcaneum, curettage, bone grafting.

$\mathrm{T}$ he aneurysmal bone cyst $(A B C)$ is an expansile cystic lesion that most often affects individuals during their second decade of life and may occur in any bone in the body. ${ }^{1-5}$ Although benign, the $\mathrm{ABC}$ can be locally aggressive and can cause extensive weakening of the bony structure and impinge on the surrounding tissues.

Jaffe and Lichtenstein first described ABC in $1942 .{ }^{6}$ As defined by the World Health Organization, the $\mathrm{ABC}$ is a benign tumor like lesion described as "an expanding osteolytic lesion consisting of blood-filled spaces of variable size separated by connective tissue septa containing trabeculae or osteoid tissue and osteoclast giant cells." 4

Address correspondence to: Dr. Prasad Soraganvi, Dept of Orthopaedics and Traumatology, Meenakshi Mission Hospital and Research Centre, Lake Area, Melur Road, Madurai- 625107, India.

${ }^{1}$ Consultant, Dept of Orthopaedics and Traumatology, MMHRC, Madurai.

${ }^{2}$ Consultant, Dept of Orthopaedics and Traumatology, MMHRC, Madurai.

${ }^{3}$ Senior Resident, Dept of Orthopaedics, DMH, Madurai.
ABCs both erode and cause 'expansion' of underlying cancellous and cortical bone. ${ }^{7}$ Around the lesion there is always a shell formed by periosteal new bone and, although this may be only millimeters thick, it prevents direct extension into the soft tissues. ${ }^{8}$ The expansile nature of the lesions can cause pain, swelling, deformity, disruption of growth plates, neurologic symptoms (depending on its location), and pathologic fracture. ${ }^{1-3}$

$A B C$ 's in the foot are uncommon. ABC's present about $1 \%$ of all primary bone tumors collectively. ${ }^{9}$ Its frequency of occurrence in foot is only about 3\% compared to other bones of body. ${ }^{10}$ Occurrence within the calcaneum are rare, and generally present as chronic heel pain and swelling, but may rarely present as pathologic fracture. ${ }^{11}$ 


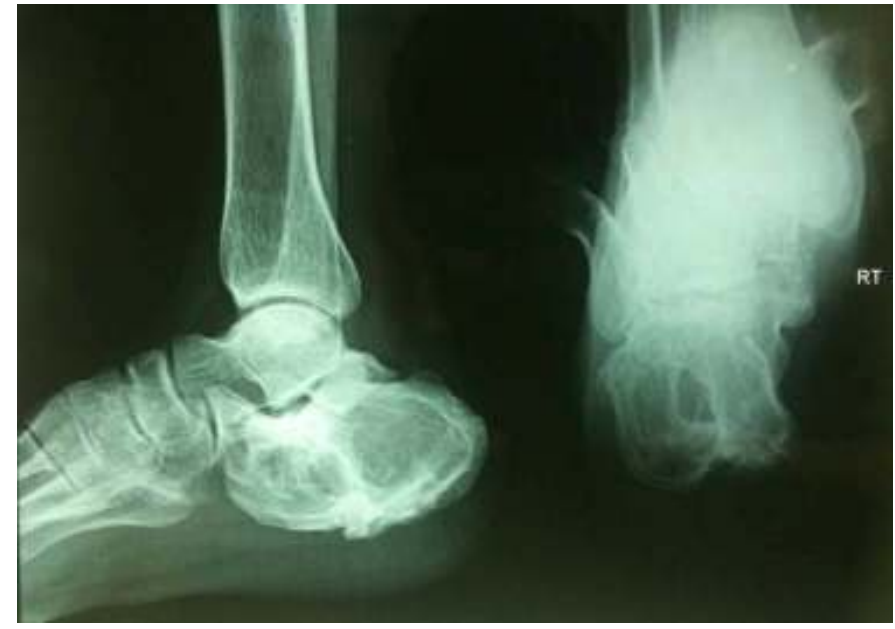

Figure 1 Pre-operative radiographs, antero posterior and lateral views showing eccentric expansile lytic lesion with thin shell of cortex and trabaculae traversing the cyst.

A plethora of cystic lesions can occur in the calcaneum, which makes definitive diagnosis difficult based on imaging only. The differential diagnosis includes simple bone cyst, ABC (primary or secondary), chondroblastoma, giant cell tumor (GCT), osteosarcoma, ossifying hematoma or pseudotumor of hemophilia. This mandates histopathological diagnosis prior to the definitive management.

We report a rare case of $\mathrm{ABC}$ involving calcaneum of 55 year-old male confirmed by histopathology report and we performed curettage and bone grafting of cyst.

\section{Case Report}

A 55 year-old male, manual laborer by occupation and known diabetic on treatment presented with a chief complaint of swelling in right heel during the last two years. An increase in swelling was associated with pain in heel from the last one year. He had difficulty in walking because of pain. For the last two months, he was unable to work due to pain. He did give history of blunt trauma prior to the onset of symptoms.
Clinical evaluation revealed swelling over the lateral aspect of the heel and the skin over the swelling was stretched. Tenderness was present on palpation but there was no local rise of temperature. The swelling was bony hard in consistency and arising from calcaneum. There were no distal neurovascular deficits or any significant lymphadenopathy.

Radiographic examination of his ankle revealed an eccentric, expansile, multiloculated lytic lesion of the calcaneum with thin trabeculae traversing the cystic cavity. (Fig. 1) There was no breach in the cortex. Based on clinical and radiological findings, a diagnosis of benign cystic lesion of right calcaneum was made.

Open biopsy of the cyst was made to confirm the diagnosis. The cyst grossly consisted of cavities filled with brown altered blood. Histopathological report revealed large blood filled cavities lined by fibrous septa, with occasional osteoclastic giant cells. (Fig. 2A and $2 \mathrm{~B}$ ) Hence the diagnosis of $\mathrm{ABC}$ involving the right calcaneum was made. The patient was scheduled for cyst curettage and bone grafting. By curvilinear incision over the lateral aspect of heel, the calcaneum was exposed. A large cortical window was made and the entire cyst curettage was done. Then the cavity was washed with saline and packed with corticocancellous bone graft harvested from both iliac crests in addition to synthetic bone substitute. The patient was advised non-weight bearing walking on the affected limb for eight weeks. Later mobilised with partial weight bearing walking for a further four weeks and then followed by full weight bearing on affected limb.

At six months of follow up, the patient was pain free and had returned to his regular activities. At two years follow-up, the patient is clinically asymptomatic. There is no evidence of recurrence. (Figs. 3A, $3 \mathrm{~B}$ and 3C) 


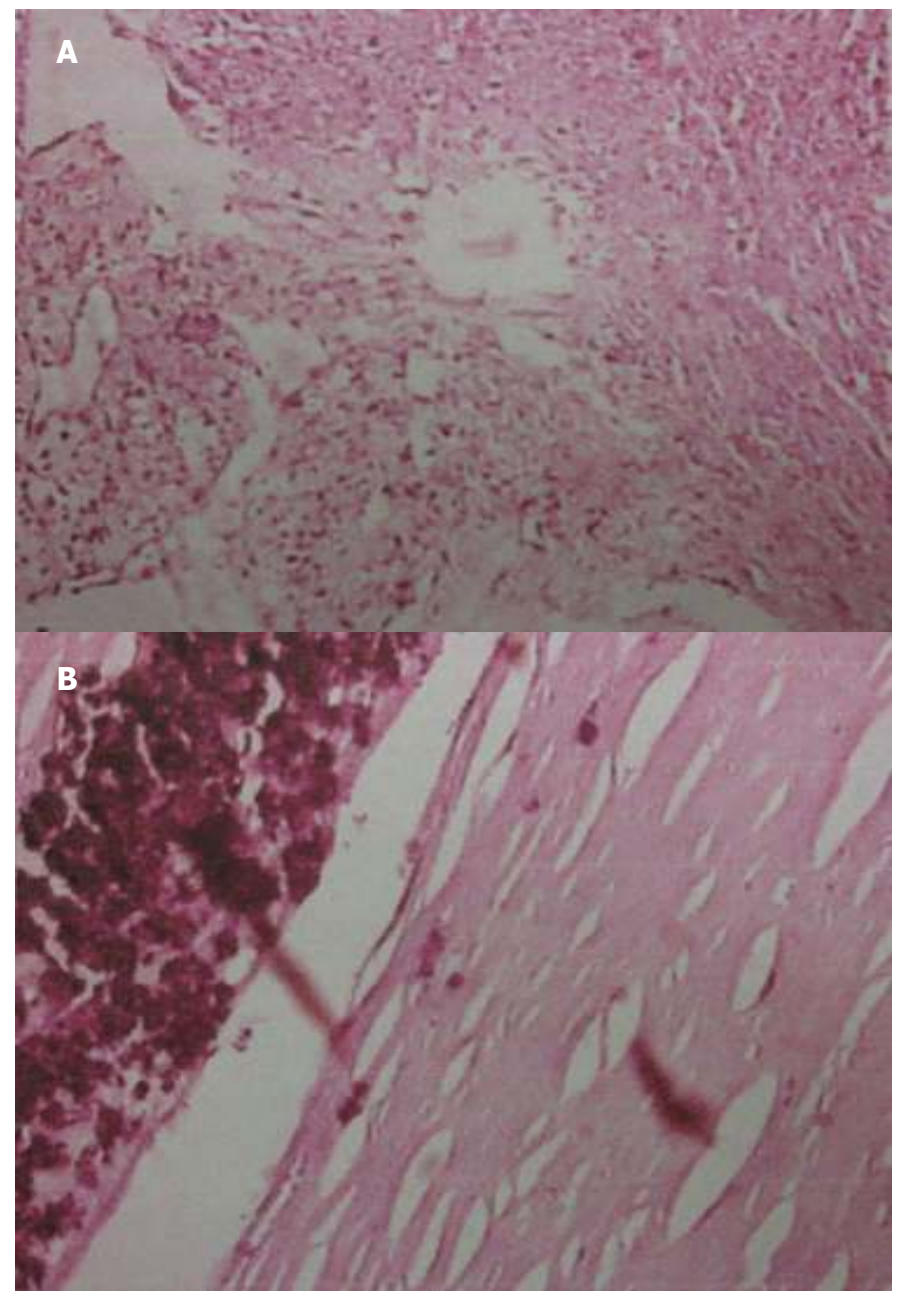

Figures 2A and 2B Histologic slides reveal large blood filled cavities lined by fibrous septa (A), with occasional osteoclastic giant cells, haemosiderin laden macrophages with a thin rim of bone. (B)

\section{Discussion}

$\mathrm{ABC}$ is an entity on its own having unique clinical, radiological and diagnostic behavior. ${ }^{7}$ The true etiology of $\mathrm{ABCs}$ is unknown. Most investigators believe that $\mathrm{ABCs}$ are the result of a vascular malformation within the bone; however, the ultimate cause of the malformation is a topic of controversy. ${ }^{12}$

The concept of an $\mathrm{ABC}$ as a secondary phenomenon occurring in a pre-existing lesion is based on the fact that in approximately one-third of the cases a preexisting lesion can be identified, the most common of which is giant-cell tumor. ${ }^{13}$
ABCs are common around the knee joint of the young $^{11}$ and have an equal incidence in both genders. About $50-70 \%$ of ABCs occur in the second decade of life, with $70-86 \%$ occurring in patients younger than 20 years, which makes this case even more unusual. ${ }^{10}$

On histology, the $\mathrm{ABC}$ is characterized by blood filled cavities lined by fibrous septa. The stroma contains proliferative fibroblasts, spindle cells, areas of osteoid formation, and an uneven distribution of multinucleated giant cells. The tissue within the septations includes cavernous channels that do not contain a muscular or elastic layer in their walls. Areas of new and reactive bone formation can also be found in the $\mathrm{ABC}$. Mitotic figures are common to $\mathrm{ABCs}$, but no atypical figures should be evident. ${ }^{10}$

Bone cysts of the calcaneum are rare lesions. These may include a wide spectrum of non-neoplastic cysts, benign or malignant neoplastic lesions ranging from simple bone cyst, ABC (primary or secondary), chondroblastoma, giant cell tumor (GCT), and an osteosarcoma (especially telangiectatic). ${ }^{11}$

Clinically, calcaneal cysts are often symptomatic and present with heel pain, although some of these lesions may remain asymptomatic and are detected as incidental findings. Even though there are many typical radiograph, computed tomographic (CT) scan, and magnetic resonance imaging (MRI) findings to confirm a diagnosis of $\mathrm{ABC}$, an open biopsy must be performed because of the high frequency of accompanying tumors. ${ }^{11}$ When a biopsy is performed, the sample should ideally include material from the entire lesion; a limited biopsy could easily cause a coexisting lesion to be missed, leaving the patient with a morbid prognosis.

There are various methods of treatment based on the site and size of the lesion, which include curettage, which may be supplemented with various adjuvant therapies such as bone grafting, use of liquid nitrogen, phenol instillation and Poly (methyl methacrylate) (PMMA) cement. 


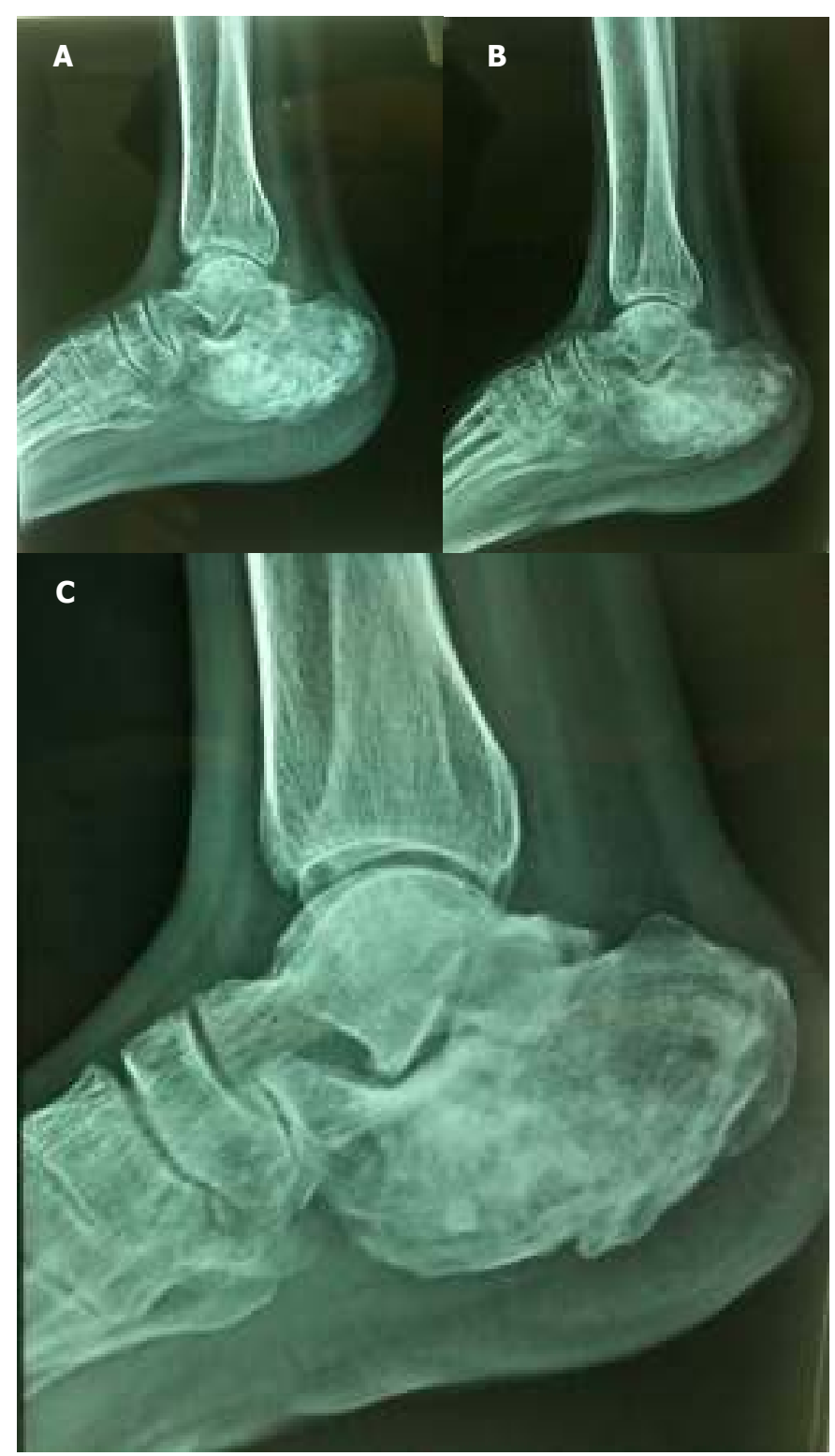

Figure 3: Follow-up radiographs showing incorporation of graft material at 1 month (A), 6 months (B), and at 2 years follow-up $(C)$ showing consolidation of graft and no recurrence.

Other modalities such as wide excision or arterial embolisation may be considered. Although relatively rare, there is no reason to assume that ABCs of the feet will respond to treatment or recur any differently from ABCs that occur elsewhere in the body. Surgical curettage is sufficient to treat most ABCs of the feet, including the calcaneum. ${ }^{14}$
Despite a favorable outcome of ABCs with an overall cure rate of $90-95 \%,{ }^{15}$ one of the most common problems encountered during management is frequent recurrence. The incidence of recurrence has been noted to vary between $59 \%$ in cases treated with intralesional excision ${ }^{16}$ and $0 \%$ in cases with resection. Recurrence usually happens within the first year after surgery, and almost all episodes occur within 2 years. ${ }^{17}$ Therefore, a patient of ABC needs to be observed for at least this period of time to exclude any recurrence. It is beneficial to detect recurrence early when the lesion is still small and easier to treat.

To conclude, $\mathrm{ABC}$ of the calcaneum is an extremely uncommon entity. Proper diagnosis entails correlating the clinical presentation, anatomical location, radiological profile, and histopathological appearance. This is imperative not only to exclude other more common histological mimics, but also for choosing the appropriate therapeutic regimen and prognosticating the disease outcome.

In a case of calcaneal cystic lesion, $\mathrm{ABC}$ should be considered as one of the differential diagnosis. Hence histological diagnosis is essential. Curettage and bone grafting is a valuable option.

\section{References}

1. Clayer M. Injectable form of calcium sulphate as treatment of aneurysmal bone cysts. ANZ J Surg 2008 78(5): 366-370.

2. Segall L, Cohen-Kerem R, Ngan B Y, Forte V. Aneurysmal bone cysts of the head and neck in pediatric patients: A case series. Int J Pediatr Otorhinolaryngol 21 2008: epub ahead of print.

3. Burch S, Hu S, Berven S. Aneurysmal bone cysts of the spine. Neurosurg Clin N Am 2008 19(1): 41-47.

4. Brastianos P, Gokaslan Z, McCarthy E F. Aneurysmal bone cysts of the sacrum: a report of ten cases and review of the literature. Iowa Orthop J 2009 29: 74-78.

5. Sun Z J, Zhao Y F, Yang R L, Zwahlen R A. Aneurysmal Bone Cysts of the Jaws: Analysis of 17 Cases. J Oral Maxillofac Surg Jan 262010 (Medline).

6. Jaffe H L, Lichtenstein L. Solitary unicameral bone cyst with emphasis on the roentgen picture, the pathologic appearance and the pathogenesis. Arch Surg 1942 44: 1004-1025.

7. Campanacci M, Capanna R, Picci P. Unicameral and aneurysmal bone cysts. Clin Orthop 1986 204: 25-36.

8. Enneking WF. Aneurysmal bone cyst. In: Musculoskeletal tumor surgery. New York: Churchill Livingstone, 1983; 1513-29. 
9. Duke Orthopaedics: Wheeless' Textbook of Orthopaedics, Aneurysmal Bone Cyst, Online article, Jan 2007.

10. Anand MK, Wang EA. Aneurysmal Bone Cyst. eMedicine, Jan 2007.

11. Unni KK, Inwards YC. Conditions that normally simulate primary neoplasms of the bone. In: Unni K K, Inwards Y C, editors. Dablin's Bone Tumors. $6^{\text {th }}$ edition. Philadelphia: Lippincott Williams and Wilkins; 2010. p. 305-80.

12. Cottalorda J, Bourelle S. Modern concepts of primary aneurysmal bone cyst. Arch Orthop Trauma Surg 2007 127(2): 105-114

13. Kransdorf MJ, Sweet DE. Aneurysmal bone cyst: concept, controversy, clinical presentation, and imaging. AJR 1995 164: 573-580.

14. Chowdhry M, Chandrasekar CR, Mohammed R, Grimer RJ. Curettage of aneurysmal bone cysts of the feet. Foot Ankle Int. 2010 31(2): 131-135.

15. Marcove RC, Sheth DS, Takemoto S, Healey JH. The treatment of aneurysmal bone cyst. Clin Orthop Relat Res 1995 311: 157-163.

16. Schreuder HW, Veth RP, Pruszczynski M, Lemmens JA, Koops HS, Molenaar WM. Aneurysmal bone cysts treated by curettage, cryotherapy and bone grafting. JBJS 1997 79B (1): 2025.

17. Rastogi S, Varshney M K, Trikha V, Khan SA, Choudhury, Safaya BR. Treatment of aneurysmal bone cysts with percutaneous sclerotherapy using polidocanol. A review of 72 cases with long-term follow-up. JBJS 2006; 88B (9): 1212-1216. 